http://jmscr.igmpublication.org/home/ ISSN (e)-2347-176x ISSN (p) 2455-0450

crossref DOI: https://dx.doi.org/10.18535/jmscr/v9i12.16

\title{
To Assess the Knowledge of MRI Safety, Among Staff Nurses and Ward Boys in Different Hospitals of Delhi/NCR
}

\author{
Authors \\ Shahjahan Ahmed (MSc RIT) ${ }^{1}$, Ruqiya Ramzan (MSc RIT) ${ }^{2}$ \\ ${ }^{1}$ Department of Radiology \& Imaging Technology, Jamia Hamdard University, New Delhi \\ ${ }^{2}$ Department of Radiology \& Imaging Technology, Jamia Hamdard University, New Delhi
}

\section{Introduction}

In today's world, MRI emerged as one of the most prompting modalities in the field of research and diagnostic imaging. MRI has an excellent imaging in diagnosis of certain diseases and lesions ${ }^{(8)}$. Growth in number of MRI scans all over the world is because of its superior soft-tissue contrast, physiological and functional applications compared with other modalities ${ }^{(5)}$.

Magnetic resonance imaging (MRI) is an established diagnostic and research tool in the field of diagnostic imaging mainly because of its excellent soft tissue delineation ${ }^{[1]}$.The very first MRI scan on human body was recorded in the year 1977. MRI has proven itself to be magnificent and multifaceted imaging technique in the field of diagnostic imaging. MRI uses magnetic and radio waves to create high resolution images of structures and organs present inside human body.

In an MRI, hydrogen atom which is present abundantly in human body act as natural magnet. When the patient is kept inside the magnetic bore of MRI, the atoms of hydrogen align themselves in parallel or anti-parallel. The alignment of atom occurs because of presence of strong magnetic field. When the RF pulse is sent, the protons are in excited state.
This produces signal which is received and analysed by computer to form images. MRI has enhanced itself by upgrading hardware and software tools with the current need. The enhancement in the hardware were made in its gradients, RF coils, magnet, transmitter, receiver and imaging technique. The three main components which comprises the MRI is:

1. Magnet

2. RF coil

3. Gradient coil

\section{Advances}

\section{Magnet}

Magnet is being one of the most significant components in an MRI. Magnet in MRI produces homogenous magnetic field. It has gone through many developments. The MRI primarily used was compact with electromagnets having very low field strength $(0.3 \mathrm{~T})$. It was later overcome with permanent magnets having field strength up to (1T). Now a days, it is equipped with super conducting magnets having field strength of $1.5 \mathrm{~T}$ and 3T. The current advancement came into existence in the year 2017 with MRI having field strength of $7 \mathrm{~T}^{(9)(11)}$. It is more than twice the previous field strengths. The FDA gave all the clearance for its clinical use in the United States. 
The 7T MRI is having its use only for neurological and musculoskeletal system ${ }^{(9)(10)(11)}$.

\section{RF Coils}

The RF coils is now facilitated with Phased Array Coils. It uses numerous coils which are having same SNR to figure a larger area.

The other progress is in the software with faster contrast scans, simplified cardiac imaging, allows MR scan of lungs, and to induce eight contrasts in single acquisition.

\section{Gradient Coils}

The gradient coils are used to detect the location of MR signal which further helps in forming images. They localize the strength in three directions ( $\mathrm{X}, \mathrm{Y}$ and $\mathrm{Z}$ axes). These all axes are present at right angle to each other. These axes in gradient coil are used for slice selection, phase encoding and frequency encoding.

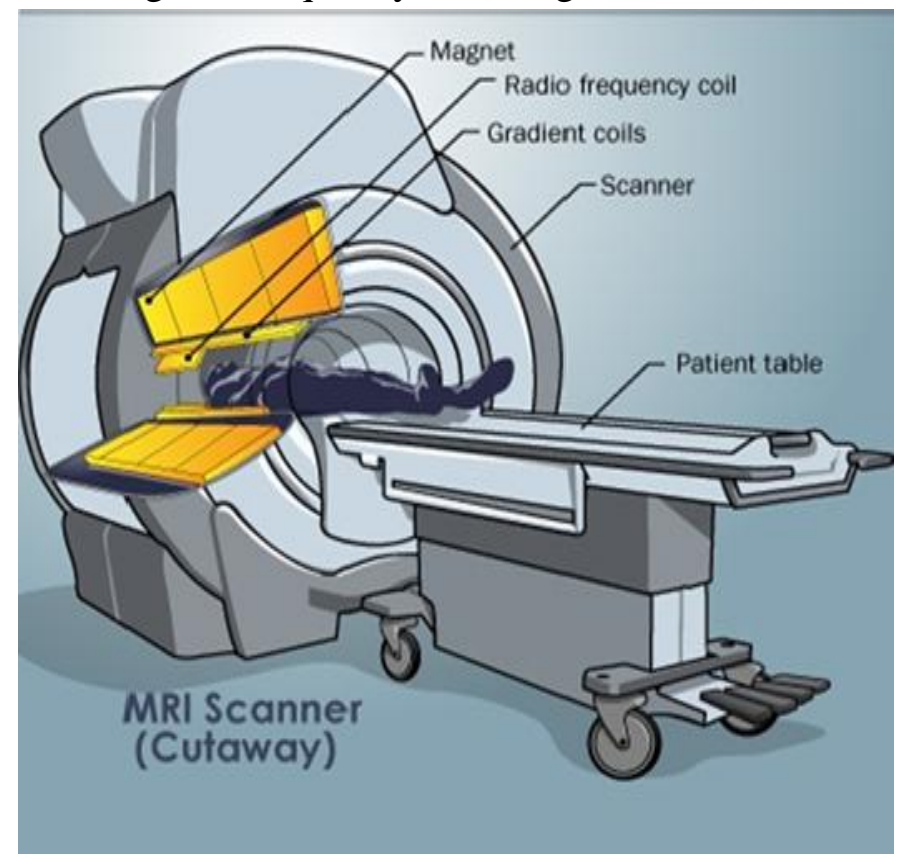

Fig 1: Components of MRI

MRI is one of the most advanced modalities in radiology department as they do not use radiation. MRI is capable of distinguishing and altering between multiple tissues using its own properties. MRI helps to assess the physiological and pathological changes in blood, its flow and CSF in discrete manner. Although MRI has diversified number of advantages compared with any other modality in the radiology department but we cannot keep aside numerous numbers of MR accidents also which in severe cases even caused death of an individual. MR examination is important but not as much as to cost a life.

Most of the MR related accidents were not reported, only lethal cases get concerned in mass media and some journals. This is the reason why no one is taking much concentration toward MR safety guidelines despite being one of the deadliest modalities if not being handled carefully. Most of the MR accidents which were reported worldwide happened because of the dereliction of duty by ward boys and illegitimate preparation of patient by nurses. After investigating the different causes of MR accidents, it is revealed that the main cause of accidents in MR environment is due to presence of strong static magnetic field which is about $>100000$ times the earth's gravitational force. The strong magnetic field draws objects composed of metals (ferromagnetic substance) and creates "flight path". Anyone who comes to the flight path might get injured and in some fatal cases can lead to death of an individual. The other causes include burn from RF coils, tangential nerve stimulation caused by alteration of gradient field.

Risk from MRI can be marked as: ${ }^{(9)}$

1) Risk from SMF.

2) Risk from RF pulse causing tissue heat.

3) Risk from varying gradient field causing incitement in peripheral nerve.

4) Risk from noise produced by MRI. This may cause short term hearing paucity.

They all may cause some of the serious risks which involves projectile effect or the missile effect, stimulation of nerve, damage to the ears causing temporary hear loss and tissue burn.

Different accidents which were reported in an MRI suite were:

- In the year 1989, patient having pacemaker lost life. It was noted that the cause of death was due to the improper functioning of 
pacemaker after having MR scan. The misfunctioning of pacemaker occurred due to MR system ${ }^{(9)}$.

- In 1992, female patient died because of pseudo information provided by the nurse to the radiographer. In the incident, the patient was equipped with intracranial aneurysm clip which was supposed to be MR friendly according to the information given by the nurse. But during the scan it was revealed that the information was not true and this led to delocalisation of the aneurysm clip during the scan resulting in death of patient ${ }^{(9)}$.

- In 1993, male patient grumble about dual vision after having MRI scan. The X-ray as well as MR scan shows occupancy of metal near patient's eye. The information was not shared by patient as he was in sedation during the MR scan ${ }^{(9)}$.

- In the year 1995, patient suffered a major bulla burn on fingers. It happened because of anchored pulse oximeter during MR scan. Burn was so severe that for treatment purpose, skin grafting was done ${ }^{(9)}$.

- In the 2001, the world witnessed one of the drastic accidents which happened because of MRI machine. A 6 years old patient named Michael Colombini lost his life because of negligence by staff nurse in New York state. The accident occurred because the nurse bought an oxygen tank inside the MRI room which got attracted by the MR machine leading to rupture the skull of boy ${ }^{(8)(9)}$.

- In the year 2004, patient with pacemaker died in UK hospital. The details about the patient undoubtly states the presence of pacemaker but the point was taken no notice of causing death of patient. The accident was similar to the one noted in 1989 where a patient with pacemaker died. MR scan led to improper functioning of pacemaker ${ }^{(11)}$.

- The capital city of India, Delhi reported its first of MRI accident in the year 2014. The accident took place when two ward boys carrying the oxygen cylinder entered the scan room and got stuck for four hours between the MR machine and metal tank $\mathrm{k}^{(11)}$.

- In 2017, in the city of Boston, patient named Paul Doherty was dragged into MRI machine along with the stretcher. The incident happened because of the negligence by staff members who took the patient inside the scan room with the ward stretcher. No major injury was reported in this case.

- Dr Ram Manohar Lohiya Hospital, Lucknow witnessed the incident in which the minister's personnel security guard entered the scan room with fully loaded gun in the year 2017. There was no risk of life in the incident but because of the missile effect it dragged the gun which led to machine $\operatorname{crash}^{(11)}$.

- The only incident which results into death of patient because of MRI in India was noted in the year 2018 in Nair Hospital, Mumbai. As the patient was on ventilator support, closed one to the patient were informed to bring the oxygen tank to the MRI room. They were also informed that the machine was OFF by the staff present in the MR room. As soon as they bought the cylinder and entered the room, one of the kin of patient got stuck between cylinder and machine that results in death.

All these accidents which were reported in MRI made the world to think about the safety practices to be followed. Several steps should be taken to lower such number of accidents.

For safety purpose the MRI suite is fractioned into four zones:

Zone I- Area which is approachable for everyone including general public, patient, hospital staff, etc. No restriction is implemented in this zone as there is no effect of magnetic field in this zone. Waiting area for public comes under this zone.

Zone II- It is the connecting zone between Zone I and Zone III. Reception area and waiting area for patient and their kin comes under this zone. No magnetic field is experienced in this zone. Patient preparation and screening is done in this area. 
Zone III- This zone is situated next to the strong magnetic field. Permission in this zone is limited to only MR personnel only and sometimes patients are also allowed only after screening. No one is allowed before screening.

Zone IV- This zone comprises strong magnetic field. Access to this room is restricted only to patient, MR personnel and medical staff only after screening. To reduce the effect of fringe field, shimming is done.

MRI is safe but sometimes it comes with great risk, not only for patients but also for other personnel who is closed to strong magnetic field or scanner"(5). "If something goes wrong, it can go very wrong" ${ }^{\prime(9)}$. The number of MR accidents are increasing with each passing day. FDA report says that MRI accident cases rises by 500\% from the year 2000 to 2009 in United States ${ }^{(9)}$. The citation of accidents in MRI has increased up to $523 \%$ from the year 2000-2009. The results were based on the reported accidents only which accounts to $10 \%$ of the total accidents occurring ${ }^{(9)}$. Most of the accidents occur because of failure and inattentiveness of an individual like clinicians, radiographers, staff nurses and ward boys ${ }^{(7)}$. Sometimes inappropriate approach also being the cause of an accident. Increasing number of accidents raised an alarm to consider patient's safety as an important aspect and it is responsibility of everyone who is directly or indirectly engaged with the profession. In radiology department, role of nurses is considered to be as important as role of a doctor, radiographer or any other staff. Nurses are the one who interconnects each staff with one another. They are the mediator who frames a relationship between a patient and the doctor/radiographer. With the help of nurses, we are able to gather more information about a patient. Nurses play crucial role in assessment of patient during examination. They are the one who ensures safety and comfort of the patient on primarily. Nurses with good vocabulary skill as well as having efficient knowledge about the modality and different procedures helps to reduce the anxiety of patient by briefing about the procedure, preparation and explanation of examination to patient and their relatives. This could somehow help to reduce the examination time and also helps to reduce artefacts which is caused due to movement by patient during ongoing of examination. Nurses also keep an eye on different monitoring devices like pulse oximeter, cardiac monitoring meter, etc. These skilled staff nurses even administer sedation and analgesia in some of the cases when they are instructed by the doctor to do so. They start or check I.V.S., assess infusers, suction patients, administer foleys and helps patients with their personal needs ${ }^{(12)}$. If a patient asks for any unconventional requisite inside an MRI suite before or during an examination, it is the nurse who informs to radiographer or technologist ${ }^{(12)}$.Nurses provide detailing of any belonging which is not permissible inside an MR room, and also about any implantation, or any type of surgery which involves metal prior to examination. They had to inform technologist or radiographer about any of the pre-medication followed by patient in ward before examination. Nurses should confirm for the personal details filled by the patient to check if the correct patient has arrived for the examination or not.

Ward boys are those who responds to the nurses and healthcare practitioners. Their main chore is to provide care to a patient, elderly person, chronically sick person or mentally or physically disabled person in a hospital. They have enough knowledge to assist patient during an examination. They help the patient in performing daily routine such as urination, bathing and dressing. They help in switching patient from trolley or wheelchair to MR scan table.

They also ensure comfort and safety of the patient just like the staff nurses. They roll the patient from one ward to another, and from different departments. They also locomote goods and vital supplies like medical equipment, linen, blood and samples.

Being a radiographer, never fully rely on information provided by nurses and ward boys. It 
is not necessary that whatever the information is provided by them is true at all time. They have different vision of thinking than ours as a radiographer. Every time ensure yourself with the information provided by the nurses and ward boys. Never compromise with the safety concern of an individual. Most of the cases which were reported happened because of inappropriate behaviour and ignorance by the nurses and ward boys. In some of the noted cases, nurses totally ignored the implantation inside a patient body despite being clearly mentioned by the referring physician. In some rare incidents, they also failed to distinguish between ferromagnetic substance and non-ferromagnetic substance that led to mis happenings.

\section{Operational Definitions}

> Projectile: Actual or near miss event where a confirmed, or potentially, ferromagnetic item is taken into the MR room ${ }^{(4)}$.

> Implants: Implant are something that is put inside human body with medical operations.

$>$ Contraindicated referrals: Referral made with unsafe implant/devices in-situ causing scan to be cancelled ${ }^{(4)}$.

$>$ Quench: Release of helium gas for demagnetisation of MR machine ${ }^{(4)}$.

$>$ Safety Issues: Any other MR safety related issue not covered.

\section{Methodology}

The Observational research approach was used to accomplish the objectives of the study. The research design was pre-Experimental design. The sample size comprises of 100 nurses and 45 wardboys from different hospitals which are located in Delhi/NCR over a period of two months. The study uses a convenience sampling technique method and the data was analysed using descriptive statistical method.

\section{Sample Size Formula for Estimation of Proportion}

$\mathrm{N}=\frac{z^{2} p q}{d^{2}}$

$\mathrm{Z}$ at $5 \%$ level of significance $\mathrm{z}=1.96$

$\mathrm{p}=$ proportion of number of correct answers $\mathrm{q}=$ proportion of number of incorrect answers $\mathrm{N} 1=100$ at $10 \%$ precession (for the nursing staff) $\mathrm{N} 2=45$ at $15 \%$ precession (for the Ward-boys)

\section{Study Design}

Acceptance for the study was obtained from the ethical committee, Jamia Hamdard University (Deemed to be University). A self-structured close ended questionnaire was prepared and justified by 3 subject experts. The respondents were briefed about the study. They were explained that being part of the study is completely voluntary, they can also opt out at any point of time during the study. The respondents reserve all the rights. They were ensured that all the information will be kept strictly confidential. All the information related to their rights are shared in the local language which they can read and understand.

Two sets of questionnaires were prepared separately for the two types of population i.e.one for the nurses and the other for the ward boys. The questionnaire for the nurses comprises of 22 questions while for the ward boys the questionnaire comprises of 12 questions. The questionnaires were prepared in local language and English. Because of the pandemic SARS Covid 19, the questionnaire was sent via online mode through Google Form and responses were collected. Respondents were asked to complete the questionnaire up to their fullest knowledge. Responses from the nurses and ward boys were scrutinized one by one for each question. All the correct responses were credited with 1 mark while incorrect answers were not credited with any mark.

Based on the responses obtained from nurses and ward boys, the respondent population was specified under 3 groups i.e., High, Medium and Low knowledge. Gross score of each individual is calculated for categorization of the population. Nurse respondents who scored more than or equal to 17 i.e., $75 \%$ were considered as High Knowledge. Scorer who scored between the range 
of 11-16 were considered as Average Knowledge. While score less than 11 i.e., less than $40 \%$ were considered as Low or Poor Knowledge. For the ward boys who scored more than 11 i.e., $75 \%$ were considered as High Knowledge. Ward boys who obtained score between range of 7-11 were considered as Average Knowledge. Those who gained score less than 7 i.e., less than $40 \%$ were marked as Low or Poor Knowledge.

Prevalence of answers to each question was computed and the population was categorized separately.

\section{Data Analysis}

Data was obtained by using descriptive statistics. The data obtained was tabulated in Microsoft Excel Spreadsheet and the analysis was done using Descriptive Statistical Method, using SPSS version 20 . The present study was undertaken to assess the knowledge of MRI safety, among staff nurses and ward-boys. The data for the study were collected through online medium and were analysed and is presented in two sections.
1. Analysis of demographic data of sample subjects.

2. Analysis of Knowledge data.

\section{Results}

The study helps to evaluate the knowledge of nurses and ward boys about MRI safety in different hospitals of Delhi/NCR. A close ended questionnaire was circulated through online mode via Google Form to nurses and ward boys engaged in different hospitals of Delhi/NCR. Total number of responses received from nurses were 100 and responses from the ward boys were 45 .

\section{Working Experience}

The population (nurses and ward boys) included in study had work experience ranging from 6 months to 25 years. Table 1 depicts the sociodemographic data of nurses which includes questions related to their gender, age, their educational qualification, working years and also about their area of working in the hospital. For the nursing population majority of the population is from the age group between 20-30 years.

Table 1: Socio-Demographic data of staff nurses

\begin{tabular}{|c|c|c|c|}
\hline \multirow{2}{*}{$\begin{array}{l}\text { DEMOGRAPHIC } \\
\text { DATA }\end{array}$} & \multirow[t]{2}{*}{ VARIABLE } & \multicolumn{2}{|c|}{ FOR NURSES(N1=100) } \\
\hline & & $\begin{array}{c}\text { NUMBER } \\
(\mathrm{N})\end{array}$ & PERCENTAGE \\
\hline \multirow[t]{2}{*}{ GENDER } & MALE & 28 & $28 \%$ \\
\hline & FEMALE & 72 & $72 \%$ \\
\hline \multirow[t]{3}{*}{$\mathrm{AGE}$} & 20-30 YEARS & 69 & $69 \%$ \\
\hline & 31-40 YEARS & 26 & $26 \%$ \\
\hline & 41-50 YEARS & 5 & $5 \%$ \\
\hline \multirow{3}{*}{$\begin{array}{l}\text { EDUCATIONAL } \\
\text { QUALIFICATION }\end{array}$} & Upto10+2/Diploma & 6 & $6 \%$ \\
\hline & GRADUATE & 85 & $\mathbf{8 5 \%} \%$ \\
\hline & POST GRADUATE & 9 & $9 \%$ \\
\hline \multirow{4}{*}{$\begin{array}{l}\text { NO. OF WORKING } \\
\text { YEARS }\end{array}$} & $>1$ YEAR & 11 & $11 \%$ \\
\hline & 1-5 YEARS & 64 & $64 \%$ \\
\hline & 6-10 YEARS & 17 & $17 \%$ \\
\hline & $<10$ YEARS & 8 & $8 \%$ \\
\hline \multirow[t]{5}{*}{ AREA OF WORKING } & Emergency Ward & 23 & $23 \%$ \\
\hline & General Ward & 50 & $50 \%$ \\
\hline & All ICU Wards & 15 & $15 \%$ \\
\hline & Orthopaedics Ward & NIL & NIL \\
\hline & Surgery Ward & 12 & $12 \%$ \\
\hline
\end{tabular}

There were 100 participants for the nursing population. Out of which $72 \%$ were female and only $28 \%$ were male. 


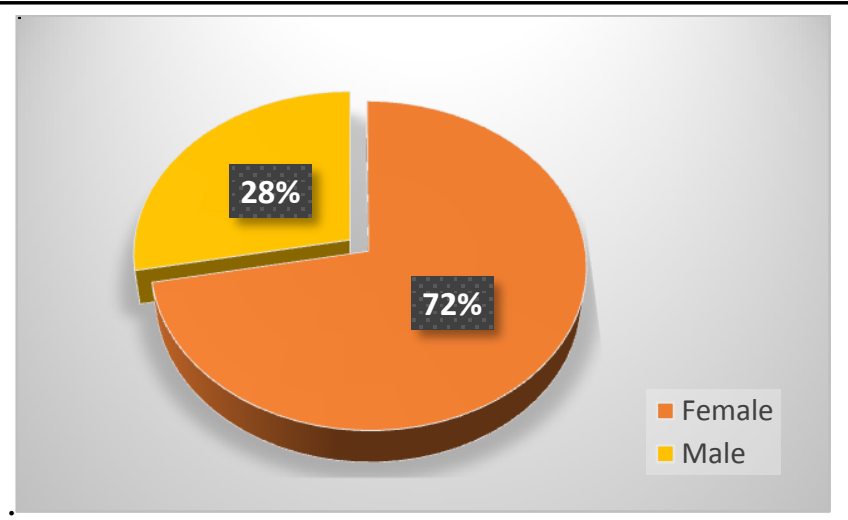

Fig 1: Gender wise distribution of nurses

Maximum number of nursing population were working in general ward of hospital.

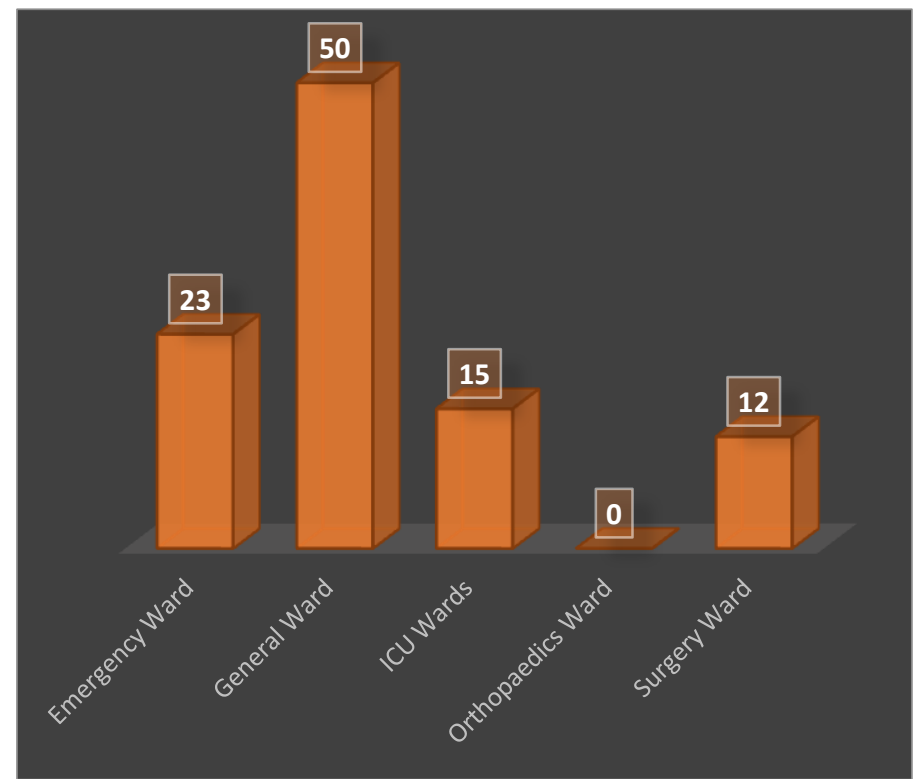

Fig: Distribution of nursing population based on their area of work

Objective 1: Assessment of knowledge on MR safety practices amidst the nurses

\section{Response of the nursing population}

Responses from the respondents were collected and specified under three categories according to the points scored by the scorer. Scrutinization of responses shows that majority of population had High Knowledge about the safety aspects that has to be followed while dealing with MRI. Almost $50 \%$ of the nursing community has High Knowledge of safe MR practices. There score falls in the range of 17-22. Around $44 \%$ of the population had an Average knowledge. There score was in the range between 1116. The population which had Low Knowledge was less. Only $6 \%$ of the population had low knowledge having score less than 11 . The average score of the respondents with a sample size $(\mathrm{N} 1=100)$ were 16.08, have range between 8-22. 


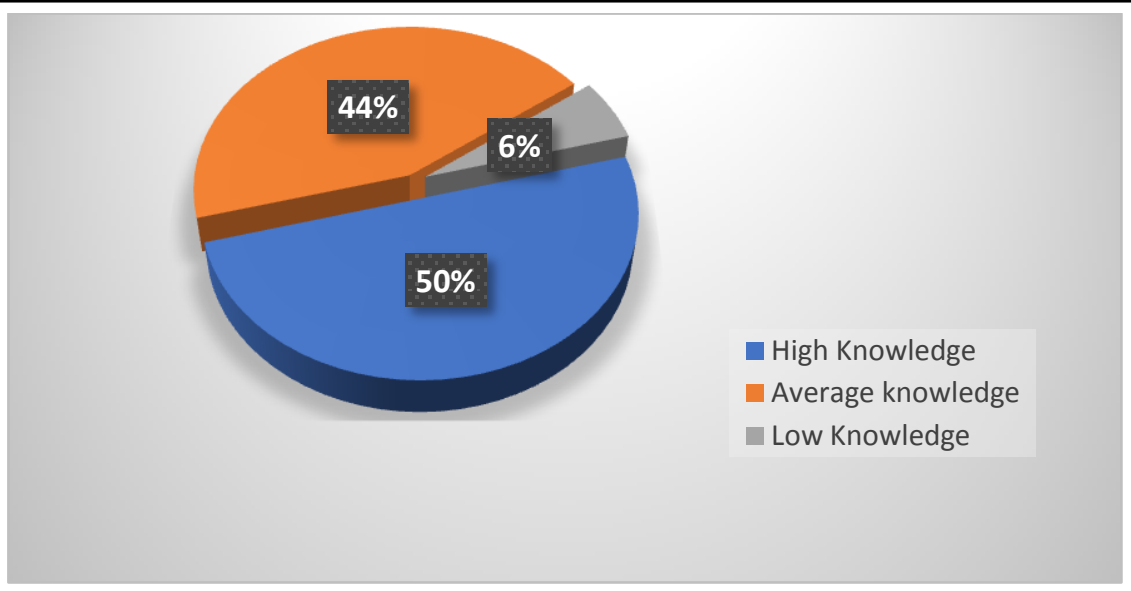

Fig 3: Response of the nurse population

From the responses by the nurse population, we can admit that majority of the population from nursing group had good knowledge about safe MR practice but while proceedings with individual question raise concern about safe MR practice.

\section{For Nursing Staff}

Table 2: Response of nurses about previous experience and training related to MRI safety

\begin{tabular}{|l|c|c|c|}
\hline & YES & NO & TOTAL \\
\hline 1. $\begin{array}{l}\text { Do you have any previous experience in Magnetic Resonance } \\
\text { Imaging (MRI) suite? }\end{array}$ & $\mathbf{7 1}$ & $\mathbf{2 9}$ & $\mathbf{1 0 0}$ \\
\hline $\begin{array}{l}\text { Have you received any training related to Magnetic } \\
\text { Resonance Imaging (MRI) safety? }\end{array}$ & $\mathbf{3 4}$ & $\mathbf{6 6}$ & $\mathbf{1 0 0}$ \\
\hline
\end{tabular}

Table 2 depicts the status of nurses about if they had any previous experience in MRI. $71 \%$ of the population had encountered with MRI modality. On questioning about if they had received any training related to MRI safety, only $34 \%$ of the population agrees that they had received some training regarding MRI. But majority of the population did not have received any training related to MRI safety. The training programme includes teaching about the protocols that has to be followed inside MRI suite or while bringing patient for MRI.

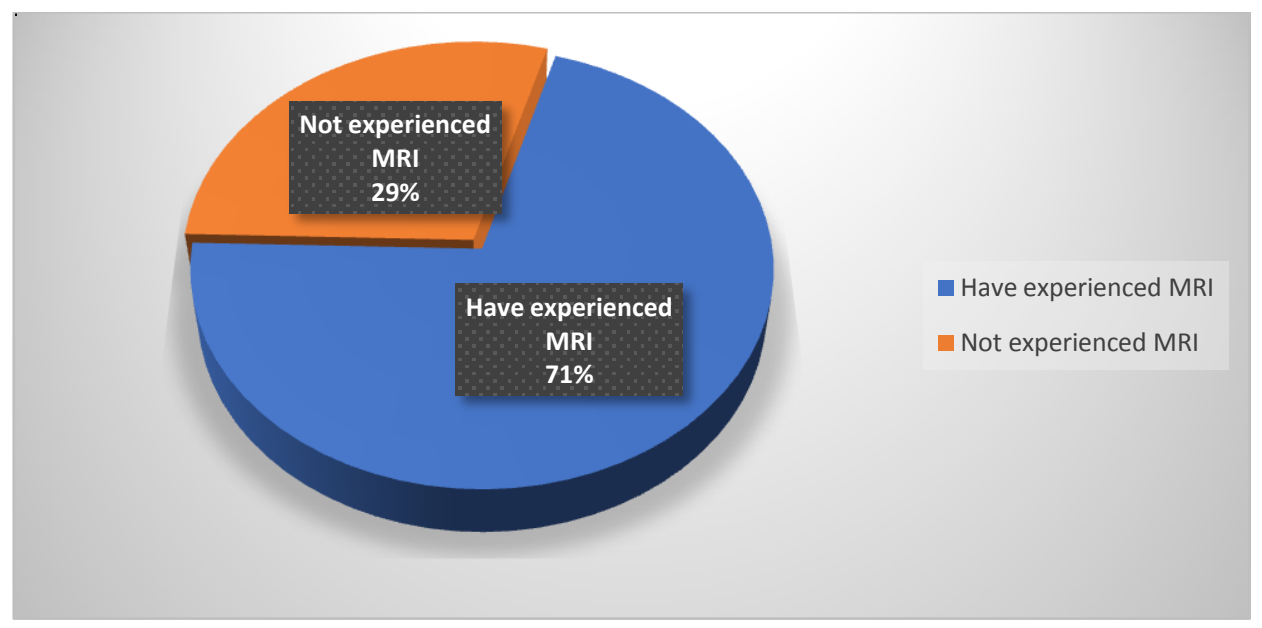

Fig: Pie chart showing nurses having previous experience in MRI

Result depicts that utmost nursing population is unaware about MRI friendly implantation present inside humans. The percentage of population which cannot differentiate between MR 


\section{JMSCR Vol||09||Issue||12||Page 80-92||December}

compatible implants is 56\%. Only 68\% of population knows that MRI uses RF pulse to send and receive signals. 69\% of the population perceives that MRI is always ON. About 78\% of the population responds that MRI is the safest modality for pregnant women after ultrasound. $89 \%$ of the nursing community were in favour of screening an individual staff before while making an entrance into the MR suite. $68 \%$ nurses admits that they cannot bring monitoring devices from wards to MRI room. $88 \%$ respondents concede the fact that they cannot take shifting devices to MRI room. They were aware that separate shifting devices were present adjacent to MRI room. It is an absolute that $35 \%$ nursing population believes that during some extreme condition, while performing resuscitation process of an individual, they can bear normal oxygen cylinder from wards. $88 \%$ nurses were attentive with the details which had to be shared with Radiographer/Technician/ Doctor while bringing patient for MRI prior to examination. 79\% population were conscious with safety gears used inside MRI like using headphones to prevent patient from permanent hear loss.

Table 4 interprets the socio-demographic data of ward-boys that contains the particulars of an individual. This encloses their gender, age, education qualification, their experience and the area of their work.

Table 4: Socio-Demographic data of ward-boys

\begin{tabular}{|c|c|c|c|}
\hline \multirow[t]{2}{*}{ DEMOGRAPHIC DATA } & \multirow[t]{2}{*}{ VARIABLE } & \multicolumn{2}{|c|}{ FOR WARD BOYS(N2=45) } \\
\hline & & $\begin{array}{l}\text { NUMBER } \\
(\mathrm{N})\end{array}$ & PERCENTAGE \\
\hline \multirow[t]{2}{*}{ GENDER } & MALE & 39 & $86.6 \%$ \\
\hline & FEMALE & 6 & $13.3 \%$ \\
\hline \multirow[t]{3}{*}{ AGE } & 20-30 YEARS & 20 & $44.4 \%$ \\
\hline & 31-40 YEARS & 22 & $48.8 \%$ \\
\hline & 41-50 YEARS & 3 & $6.6 \%$ \\
\hline \multirow{3}{*}{$\begin{array}{l}\text { EDUCATIONAL } \\
\text { QUALIFICATION }\end{array}$} & Upto10+2/Diploma & 38 & $84.4 \%$ \\
\hline & GRADUATE & 7 & $15.5 \%$ \\
\hline & POST GRADUATE & NIL & NIL \\
\hline \multirow[t]{4}{*}{ NO. OF WORKING YEARS } & $>1$ YEAR & 2 & $4.4 \%$ \\
\hline & 1-5 YEARS & 19 & $42.2 \%$ \\
\hline & 6-10 YEARS & 17 & $37.7 \%$ \\
\hline & $<10$ YEARS & 7 & $15.5 \%$ \\
\hline \multirow[t]{5}{*}{ AREA OF WORKING } & Emergency Ward & 16 & $35.5 \%$ \\
\hline & General Ward & 11 & $24.4 \%$ \\
\hline & All ICU Wards & 7 & $15.5 \%$ \\
\hline & Orthopaedics Ward & 4 & $8.8 \%$ \\
\hline & Surgery Ward & 7 & $15.5 \%$ \\
\hline
\end{tabular}

There were 45 participants in ward-boys population. The population comprises $86.6 \%$ male and $13.3 \%$ female.

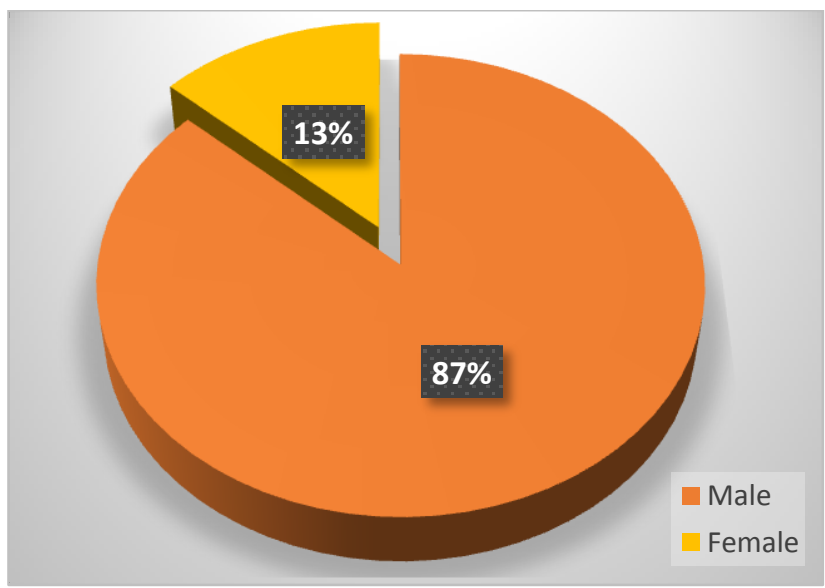

Fig 2: Gender wise distribution of ward-boys 
More number of ward-boys are engaged in the emergency ward or hospital as there are lots of patients who came to hospital in some serious conditions. They need assistance for shifting of patients as they are ill.

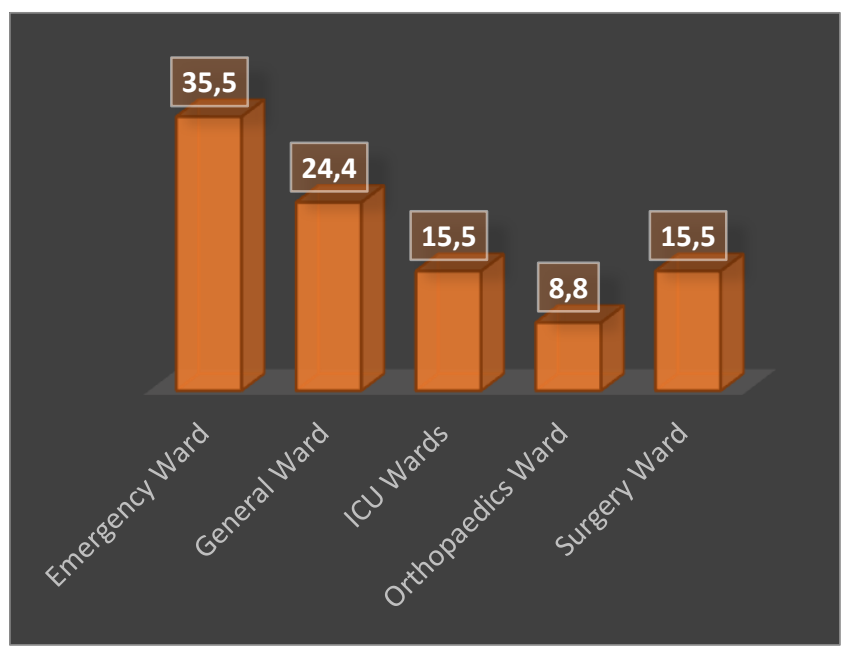

Fig: Distribution of ward-boys population based on their area of work

Objective 2: Assessment of knowledge on MR safety practices among ward-boys.

\section{Response of ward-boys}

The response from the respondents were specified under three categories after calculating their score. The result illustrates that majority of population from ward-boys had an average knowledge about safety aspects in MRI. Respondents under category of High Knowledge are few. Only $9 \%$ of the population scored points in the range of 12-14.
Major population comes under the category of Average Knowledge with score ranging between $7-11$. These constitutes $71 \%$ of the total population of ward-boys. About $20 \%$ of wardboys had Poor Knowledge of MRI safety aspects. There score was less than 7. The average point scored by ward-boys were 8.63 with range between 2-12.

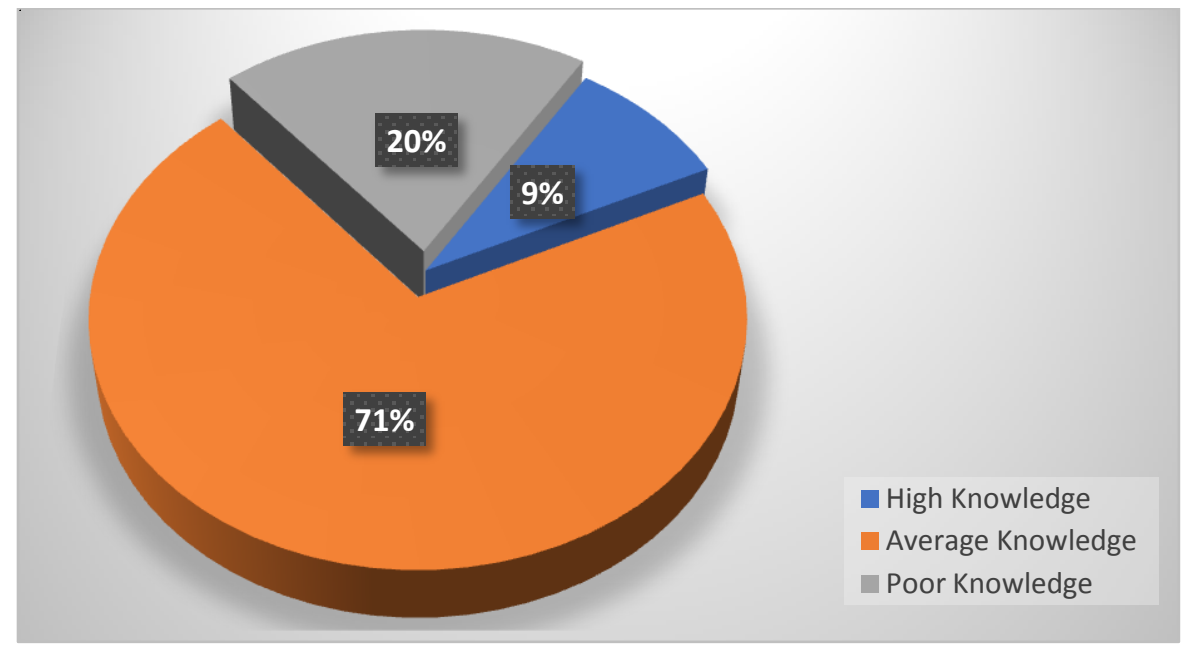

Fig 4: Response of the ward-boys population 
For Ward Boys

Table 4: Response of ward boys about previous experience and training related to MRI safety

\begin{tabular}{|c|c|c|c|}
\hline & YES & NO & TOTAL \\
\hline $\begin{array}{l}\text { 1. Do you have any previous experience in Magnetic Resonance Imaging } \\
\text { (MRI) suite? }\end{array}$ & 38 & 7 & 45 \\
\hline $\begin{array}{l}\text { 2. Have you received any training related to Magnetic Resonance Imaging } \\
\text { (MRI) safety? }\end{array}$ & 6 & 39 & 45 \\
\hline
\end{tabular}

Table 4 shows the responses from ward-boys. Maximum population had previous experience in MRI suite. They help in shifting patient especially those who are weak and in critical condition. $84 \%$ of population is familiar with MRI while only $16 \%$ population had never encountered with MRI. Only $13 \%$ of the population had gained training related to
MR safety. $87 \%$ of the respondents from ward-boys did not have any training that raises an alarm toward patient's safety. These are the one who encounters with patient shifting, shifting of trolley, crash carts and in some emergency condition also the cylinders outside the MRI room.

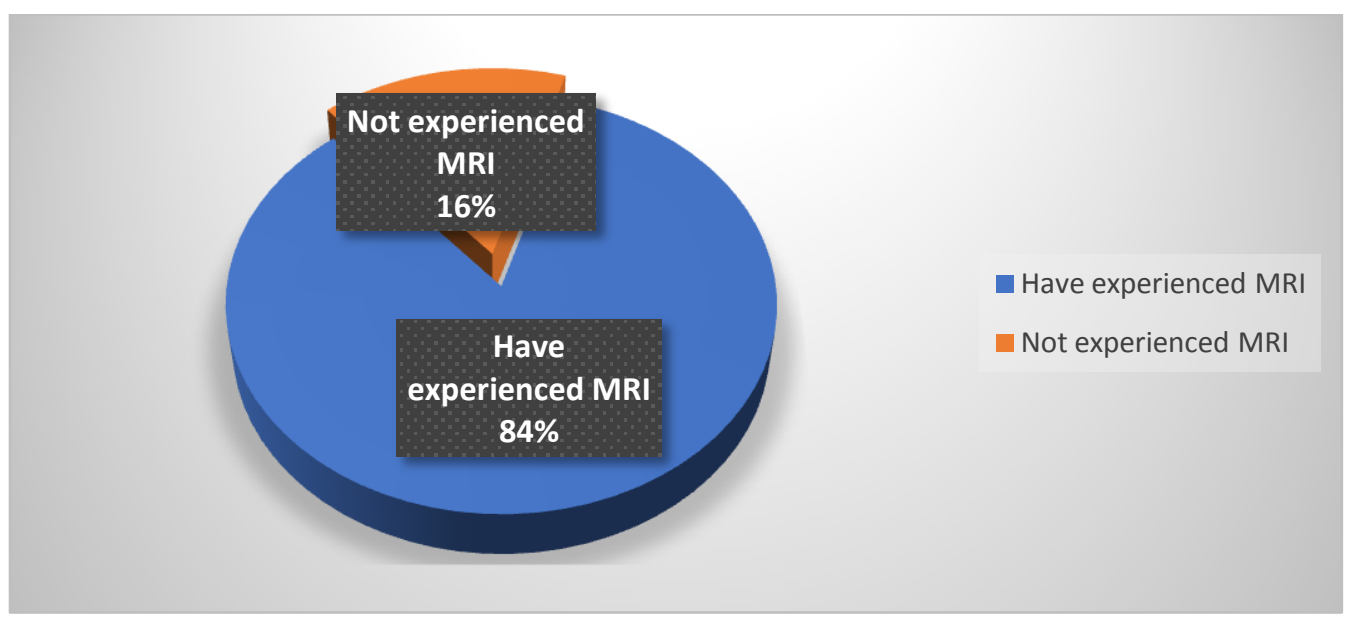

Fig: Pie chart showing ward-boys having previous experience in MRI

Responses from ward-boys reveals the fact that $53.3 \%$ of population is unaware that magnetic field is always $\mathrm{ON}$ in an MRI. $62.2 \%$ of population is unfamiliar with MRI friendly implants. $55.5 \%$ of respondents does not have knowledge about the different zones present in MR suite. $51.1 \%$ of the ward-boys population does not know about screening of patient is important prior to MR examination. $60 \%$ of the population was conscious that MRI is safe for pregnant women. $66.6 \%$ of population delineate about the objects to be removed from patient body prior to examination. $86.6 \%$ of ward-boys acknowledge screening of staff person before entering MR suite. $86 \%$ of respondents knows that they cannot bring patient on normal shifting devices like trolleys and wheel chairs from wards.

\section{Discussion}

MRI has an excellent imaging in diagnosis of certain diseases and lesions. An increasing demand of MRI day by day, numerous incidents of accidents have been reported in India as well as worldwide. The various accidents reported till now is because of the strong static magnetic field, projectile accidents (flight path), and mainly because of negligence. Most of the cases were not reported, only some of the lethal cases were reported. Various studies were previously done in India and world-wide amongst the cases but limited studies were conducted to find the root cause of such accidents while having MRI. The FDA report says that MRI accident cases rises by $500 \%$ from the year 2000 to 2009 in United States. The citation of accidents in MRI has increased up to $523 \%$ from the year 2000-2009. 
We undertook this study to Assess the Knowledge of MRI Safety, Among Staff Nurses and Ward Boys in Different Hospitals of Delhi/NCR.

\section{Demographic Characteristics}

In my study the sample size for nurses consists 100 samples out of which $69 \%$ were female and were between the age group of 20-30 years. A similar study was conducted in University Hospital in city of Sao Paulo by Marcos Souza Lima et al. The study was descriptive and time based (three months duration) to reviewed about the knowledge of nurses about MRI safety. The participating nurses in the study were mainly female i.e., $79 \%$ having an age group between 22 30 years $^{(3)}$.

For the study a self-structured closed ended questionnaire was used for collecting data. In previous studies technician were respondents for the survey and also some of the studies were done on the number of previous accidents that were reported. My study was based on the nursing population and ward-boys population that makes a difference from rest of the previous studies. I took this population as this population is the one who is primarily involved with the patient having MR examination. And also because of the previous studies reveals the fact that majority of accidents happened due to negligence by nurses and wardboys.

\section{Knowledge Regarding MRI Safety}

The current study shows overall good response from the nursing staff. Majority of population i.e.; $50 \%$ had good knowledge and $44 \%$ had an average knowledge. while dealing with individual questions from the questionnaire it is revealed that there is lack of knowledge about MRI basics. About $56 \%$ of population were not conscious about MR friendly implants. They even don't have the knowledge about life saving protocols to be followed in MRI room if a patient finds difficulty in breathing or if having cardiac arrest. 32\% agrees with taking monitoring devices from wards to MR room.
On the other side, ward-boys population had an average knowledge i.e., 71\%. The individual question shows that this population is also unaware about permissible implants or metals. They don't know that before making entrance to the MR room patient as well as staff had to pass through metal detector door or have to be screened with hand handled metal detector.

The results from both the population raise an alarm toward the safety of patient. Some of the responses show negligence from staff like on asking if they could bring ferromagnetic projectile to MR room, there response was that they could bring the trolleys, wheel-chairs and stretchers directly from ward to MR room.

The study by Darren Hudson et.al., shows that $32 \%$ of the accidents took place because of the contraindicated referrals including pacemakers, braces, neuro-stimulators and other implants. The study also shows that $21.5 \%$ of the population had issues with screening prior to enter MR room ${ }^{(4)}$. The current study also shows that there were only $42 \%$ of the population were aware about the contraindicated referrals including implants. Rest of the population were unaware about the contraindicated referrals of MRI. Only $11.7 \%$ of population had issue with the screening prior entering to MRI.

The study by Disha Faujdar reveals that $8 \%$ of the nurses were not clear and thinks that its normal to send patients with pacemaker, aneurysm clips and stents for MRI. The current study depicts that $14 \%$ of the population were not sure with fact that pacemaker, aneurysm clips and stents are completely contra-indicated for MRI. $16 \%$ of the nurses lack knowledge about the contrast used in MRI. The current study shows that $25 \%$ of nurses were unaware about the contrast used in MRI. $50 \%$ of nurses thinks that MRI is not safe for the pregnant women. In the current study the population is more aware that MRI is safe for pregnant women. The percentage is reduced to $22 \%$ nurses who believes that MRI is not safe for pregnant women $^{(8)}$. 
The current study shows that the main cause of accidents in MRI is lack of knowledge about contraindicated referrals resulting into projectile (flight path) accidents. Similar findings were reported in other studies also where the main cause of accidents were due to projectiles that are not permissible and completely contraindicated in MRI.

The study shows that there is need for proper training amidst nurses and war-boys. There is need to educate the staff about basics of MRI as this would be helpful in decreasing the cases of accidents.

\section{Conclusion}

After numerous studies based on the topic related to MRI safety, it is revealed that most of the accidents happened because of the negligence and inattentiveness behaviour of the workers. a large number of accidents can be prevented if they had proper knowledge. There must be workshops and seminars organized for the staff especially for nurses and ward-boys about caring and safety of patients and also about the safety protocols to be followed while going for radiological examination once in a month. Safety related posters indicating the contraindications (like coins, ATM cards, pins, keys, etc.) for MRI must be displayed. A safety officer must be employed to regularize safety protocols in Radiology Department and also to ensure safety for patients as well as staff at the ground level $^{(9) \text {. }}$

Keywords: MRI, safety, nurses, ward boys, knowledge, lethal, amidst, switch, apart

\section{References}

1. Jacobs MA, Ibrahim TS, Ouwerkerk R. MR imaging: brief overview and emerging applications. Radiographics. 2007 Jul;27(4):1213-29.

2. Shellock FG, Crues III JV. MR safety and the American College of Radiology white paper. American Journal of Roentgenology. 2002 Jun;178(6):1349-52.
3. Lima MS, Shigueoka DC, Salvador ME, da Silva Canteras LM, Carmagnani MI, Ajzen SA. Assessment of nurses' knowledge about magnetic resonance imaging in a university hospital in Sao P aulo. International journal of nursing practice. 2012 Dec;18(6):574-81.

4. Hudson D, Jones A. A 3-year review of MRI safety incidents within a UK independent sector provider of diagnostic services. BJR Open. 2019 Jan 1;1(1).

5. Mozammel Hossen., Sohel Rana, Tanzila Parvin, SM Muraduzzaman, Dr.Mustaque Ahmed. Evaluation of Knowledge, Awareness, and Attitude of MRI Technologists towards MRI safety in Dhaka city of Bangladesh". International Journal of Pure Medical Research. 2020, 5.5

6. Goolsarran N, Zarrabi K, Garcia C. Using a resident-led patient safety quality council to educate future QI leaders. Medical Education Online. 2021 Jan 1;26(1):1855699.

7. Nicholas J. Ferris., et al" The 2005 Australian MRI Safety Survey". American Journal of Roentgenology. 188.5 (2007): 1388-1394

8. Disha Faujdar., et al. "Knowledge of Magnetic Resonance Safety Measures among Ward Nurses and Housekeeping Staffs in Tertiary Care Hospital". EC Nursing and Healthcare 2.11 (2020): 67-72

9. Ng KH, Ahmad AC, Nizam M, Abdullah B. Magnetic resonance imaging: Health effects and safety. In Proceedings of the international conference on non-ionizing radiation at UNITEN (ICNIR2003) electromagnetic fields and our health 2003 Oct 20.

10. https://www.itnonline.com/article/recentadvances-mri-technology

11. https://science.howstuffworks.com/mri.htm

12. https://nursejournal.org/careers/radiologynurse/

13. https://pubmed.ncbi. nlm.nih.gov/10139086/ 